\title{
Ethics of science communication on the web
}

\author{
Maxine Clarke* \\ Nature, Nature Publishing Group, Macmillan Building, 4 Crinan Street, London N1 9XW, UK
}

\begin{abstract}
Scientists have evolved a unit of communication to describe their new results and findings: the peer-reviewed scientific paper. The internet is full of erroneous and even dangerous information that is difficult for people without a scientific education or training to interpret in context, particularly given the uncertainties inherent in the scientific process. Those interpreting science for the public, whether journalists, educators or other communicators, should use peer review as a benchmark.
\end{abstract}

KEY WORDS: Publishing $\cdot$ Science $\cdot$ Peer review $\cdot$ Internet $\cdot$ Research Resale or republication not permitted without written consent of the publisher

\section{BACKGROUND}

Scientific research is, by its nature, technical and uncertain, and it is this combination which poses special problems for its communication. Scientists have always communicated informally: in their places of work, among other scientists working on similar problems, at conferences, in 'journal clubs' discussing publications and in the form of drafts and 'preprints' of manuscripts describing their results.

When it comes to formal communication, scientists submit a paper describing a body of their research to a journal. Editors make an assessment of the suitability of the manuscript for that particular journal and seek a technical opinion from independent peer reviewers, i.e. other experts in the field who assess the results and the argument for their scientific soundness (see the Nature journals peer-review policies at www.nature. com/authors/editorial_policies/peer_review.html and the Nature peer-review debate at www.nature.com/ nature/peerreview/debate/index.html). If the review indicates that there are no technical problems, the paper can be published if it meets the editorial criteria of the journal. If the paper is declined, its authors can strengthen it (or not) and submit it to another journal, where the process is repeated.
A research paper published in this way is formally recognized as a unit of output for its authors, unlike many of the informal ways by which scientists communicate their research. The peer-reviewed paper is an honest account of a piece of work, not overselling its conclusions and, ideally, written in a comprehensible manner. Once the paper is published, it is in the public domain: fair game for comment by journalists and other commentators; and fair game for interpretation that anyone may put on it, whether that person might be genuinely trying to understand the work or whether they are 'quote mining' to prove a preconceived point.

\section{SCIENCE ON THE WEB}

\section{Embargo policies}

Over time, scientific journals have evolved systems to assist the communication of research results to the public in an ethical way. Most, if not all, have embargo policies like that of Nature, the journal for which I am an editor, in which authors must not discuss material submitted to the journal with the media until it is formally accepted for publication. After acceptance, authors can discuss their work with the media a week 
or so before the publication date, under embargo conditions (see www.nature.com/authors/editorial_policies/ embargo.html). The Nature journals believe that their embargo serves scientists, authors, journalists and the public. Our policy is to release information about our content in a way that provides fair and equal access to the media, allowing it to provide informed comment based on the complete and final version of the paper that is to be published. Authors and their institutions' press offices are able then to interact with the media ahead of publication, and benefit from the subsequent coverage.

Peer review is a means of giving journalists confidence in new work published in scientific journals. Premature release of research results by scientists to the media denies journalists that confidence. It also removes journalists' ability to obtain informed reactions about the work from independent researchers in the field.

Although not all journalists and not all science bloggers (who write about scientific research on the internet) are supportive of this type of policy, this system of scientific communication has evolved for several reasons and serves multiple purposes for scientists themselves as well as their employers and funders. Putting some of these to one side, I want to concentrate on the twin elements of technical complexity and uncertainty that I feel are at the core of the question of the ethics of science communication: many people in the world do not have a high level of scientific education and are not trained in the style of critical thinking necessary to evaluate research results. An understanding of statistics, for example, is essential for the appreciation of almost every discipline of science. Yet relatively few people are aware of even the basics of statistical power and the assessment of probabilities.

\section{The scientific record}

One reason why scientists have evolved the system of journal publication is that scientists use journals to provide a 'quality stamp' for their research, so that people can take as read the conclusions without having to understand the details. Scientists in a discipline write the papers and others in the field peer-review and edit them, with the result that a reliable, archived and indexed record of research conclusions and directions (managed and archived for them by the journals) is built up. Scientists understand also that these papers are not necessarily 'right'. The peer review process ensures that the technical underpinnings of a piece of work are correct as far as can be judged (unless there is some deliberate fraud or other misconduct, or an honest error such as an equipment malfunction), but interpretation on the basis of results or calculations is on the balance of probability, not a certainty. Interpretations can, in the light of subsequent work, turn out to need refining or re-thinking, or to be wrong. But this hindsight does not mean that there was anything wrong with the published paper, which is a record of what was best known at the time. The thread of logic from paper to paper provides a wonderful history of thought which is captured in literature reviews or books, for example, which show how concepts arise and are developed, blind alleys and all.

Understanding this process is less easy for those who are not scientifically trained. To learn about the world's knowledge, teachers, journalists and other communicators who do have a scientific education seek to explain the output of research to those who lack the tools to comprehend the details of every technical paper that is produced (sometimes by authors who have spent many years of their professional lives thinking about that one question). Insofar as science is an explanatory process, this system works pretty well. It is when we turn to uncertainty that difficulties arise, because many people believe that uncertainty is intrinsically a bad thing - that it means that the scientific conclusion is incorrect, or 'spun' in some way.

\section{The internet}

The internet exacerbates these difficulties of science communication. We all know of areas of science that are susceptible to controversies: among the most wellknown are climate change and the genetic modification of crops and other organisms. In the former case, many people do not understand the concept of a computer model and its limitations or that weather is a local process, whereas climate is a global one, subject to different physical forces. It is this combination of a lack of technical understanding and an unrealistic expectation that science is absolute, rather than about uncertainty or prediction, that causes very basic confusions. Add commercial and political interests to the mix, whether the oil, agriculture or tobacco industry, and one can see how confusions can escalate into paranoia, manipulations of the truth and conflict of all kinds. The losers in this mess are the people who are trying to understand our natural world, whether those people are the researchers themselves, people in other professions, students, or anyone.

The internet can be a powerful disruptive force, as anyone who has seen 'vested' and 'hidden' interest forums can attest. But the internet is a medium, not a message in itself. There are plenty of excellent web sites in which scientific information is being archived, discussed and explained, and which are used by scien- 
tists as research tools or by anyone. And 'anyone' includes people and organisations that use scientific research to do their work, for example policymakers, medical doctors, insurers or aeroplane designers. Scientific communication is not a 'nice to have' but is an essential fuel of almost every part of the global economy.

Although there are many 'crank' science websites, there are also many reliable, useful resources, which can be educational or single-issue-based. The worst of the crank theories that spring up on the internet (for example the anti-vaccination sites discussed in a recent Nature Immunology editorial, see www.nature. com/ni/journal/v9/n12/full/ni1208-1317.html) are often quickly countered by specific rational counterparts: there are very good HIV information websites, for example, initially set up as responses to 'AIDS denial' pseudoscience claims but since developed into useful, independent resources (e.g. AIDSinfo from the US government, www.aidsinfo.nih.gov/). There are plenty of sites and blogs that debunk others promoting medical 'cures', paranoia and other hokum. Some of these sites are quite systematic, for example www.realclimate.org, in which climate scientists provide regular, balanced and clear accounts of research for non-scientists (and other scientists) without any associated media, political or industrial 'spin' - the goal is to tell the science like it is, including the degree of uncertainty, so far as can be ascertained.

\section{What to trust?}

How are people to know what information to trust on the internet? Scientific research papers are necessarily obscure to almost everyone-even the well-written ones are extremely technical and are a distillation of deep thinking. Despite facile arguments about 'taxpayers' dollars mean taxpayers must have access', as if a scientific paper is equivalent to one's rubbish being removed, the truth is that most scientific papers are hard even for researchers in related disciplines to understand fully. However, the question goes far beyond the limited one of whether people are able to read the actual text of a paper and understand its implications and, just as important, its limitations: as far as the 'internet-reading public' is concerned, the interpreters of science can be more significant than the scientists themselves. And this is a considerable ethical challenge, as there is no peer review for the interpreters - whether journalists, or scientists and others writing on blogs (a medium that usually does not have the safety-net of an independent editor).

If someone does not have a scientific education or scientific knowledge, and is more familiar with a per- suasive or emotion-arousing style of argument than a critical, logical one, how is this person to tell the difference between objective and slanted information? The context and implications of work described in the scientific literature are usually not apparent to nonspecialists, whether scientists in other disciplines or a lay audience.

There are many freely available, excellent scientific news websites and blogs which report and analyse peer-reviewed research, some of which use a tool called Research Blogging (e.g. www.researchblogging. org). Many of these sites are interactive, so that users can ask questions and otherwise comment. There are good online reference sources, though free ones such as Wikipedia (www.wikipedia.org) are more attractive to most people than subscription reference sources, which may be more accurate.

We can use Google and other search engines to discover information about anything on the internet, but our search skills probably do not match our lack of patience and need to know things fast-after all, that is why most people turn to the internet: to find out some information quickly and conveniently without having to go to a library to consult the appropriate printed reference book. How much time will that person spend to find the most reliable, as opposed to the first, source? How quickly will the searcher be overwhelmed and hastily pick out an answer? Many of the comment threads even on good scientific journal and other science-oriented websites sometimes cause me to despair at the amount of ignorant and indulgent drivel that is written: surely a discouragement to sensible experts who might otherwise contribute, not least because of everyone's time constraints.

\section{Access}

Scientists themselves are not a homogeneous collection of people who all see things the same way or use the same style of logic. Some scientists and exscientists are prime movers in promoting disinformation on the internet, and some journalists are keen to promote causes or angles for their own reasons that have nothing to do with 'pure' science communication. Many scientists have commercial and other vested interests, or strong political ideologies, rather than being dedicated to objective interpretation. Other scientists, mainly young ones perhaps on short-term employment contracts, are frustrated at their perception of narrow career options available to them and become irritated at what they see as a 'closed' journal publishing system, and vent their spleen online. The 'open access' publishing model is a favourite topic for hasty and callow statements, in which the cost and 
effort of publishing high-quality research is underestimated and, increasingly, attacked. Yet whether a paper itself is published under an 'author pays, anyone can read' model or a more standard 'author publishes for free and libraries or individuals subscribe' reading model is not relevant to the quality of the research or how it is interpreted. Furthermore, arguments about access to the scientific literature have spread into irrelevant areas. The very peer-review system itself is under threat by unsubstantiated claims that the system is biased, whether in favour of established researchers or theories and against innovation; or in favour of 'headline-grabbing' conclusions and against solid but unglamorous research (take your pick from a range of contradictory criticisms!). Yet peer-review is the one measure of scientific quality that has been demonstrated to work, by the simple but self-evident fact that the vast majority of published science is reproducible and is built upon. Some detractors point to reports of minor errors that are missed by the peer-review and editing process, but any study of the quality of the peer-reviewed literature needs to compare the published version of papers with the un-peer-reviewed versions that were submitted to the journal.

At one extreme, the dissenters argue that all research should be published immediately on the internet, as soon as the draft is written, and all should have access to it. Great importance is attached to instant availability of data, little to the quality of interpretation and explanation. Advocates of this 'speed is all' approach argue that the scientific community, and the world at large, will (in an undefined process) select what is good and ignore what is not. Although this type of communication may work very well between scientists using the same type of data to work on similar problems, if there are neither scientific journals and a 'unit' of publication, nor knowledgeable interpreters of this information, the vast majority of people will not understand a substantial amount of scientific output, know what is reliable, or

Editorial responsibility: Stephanie Suhr-Sliester, Oldendorf/Luhe, Germany be able to filter out what is of use or interest to them. On the internet, everybody is king or queen for the time that he or she is interacting with it, especially on 'social' websites where online commenting and blogging occur without any constraint other than selfrestraint. How can people know what to believe? Are they going to believe claims because they are made by 'a scientist'? Are they going to rely on a journalist's understanding of what type of science is robust and what is not; a journalist under his or her own imperative to produce newsworthy but slanted copy in an increasingly competitive publishing environment? Or will bloggers and other internet interpreters do the job?

\section{CONCLUSION}

There are no easy answers to these questions. Taken together, the high general expectation of 'getting answers instantly' on the internet, the intrinsic uncertainty of science, and the technical complexity of much of it are great challenges to scientists when it comes to explaining what they do, why it is important and how exciting it is. Perhaps there is no systematic solution to the question of how scientific research can be accurately and clearly presented and trusted, despite technical and personal tools such as aggregation, voting and ranking, tracking indices, or 'quality stamps' (such as an icon on a blog post to indicate the posting is about a peer-reviewed paper). Scientists need to decide if the priority is to focus on discovering how our natural world works and to communicate the excitement of that process, or whether they prefer the world to see them as in-fighting about processes and 'hyping' their work to attract public attention and investment. For the first of these approaches, peer-review is our best bedrock, on which interpreters can build. The second approach can only allow a non- or anti-scientific environment to flourish.

Submitted: November 14, 2008; Accepted: November 19, 2008 Proofs received from author(s): November 28, 2008 